\title{
\begin{tabular}{l|l} 
Mitraries & DSpace@MIT
\end{tabular}
}

\author{
MIT Open Access Articles
}

\section{On the measurement of intensity correlations from laboratory and astronomical sources with SPADs and SNSPDs}

The MIT Faculty has made this article openly available. Please share how this access benefits you. Your story matters.

Citation: Schroeder, Edward, Philip Mauskopf, Genady Pilyavsky, Adrian Sinclair, Nathan Smith, Sean Bryan, Hamdi Mani, et al. "On the Measurement of Intensity Correlations from Laboratory and Astronomical Sources with SPADs and SNSPDs." Edited by Fabien Malbet, Michelle J.

Creech-Eakman, and Peter G. Tuthill. Optical and Infrared Interferometry and Imaging V (August 4, 2016).

As Published: http://dx.doi.org/10.1117/12.2233536

Publisher: SPIE

Persistent URL: http://hdl.handle.net/1721.1/110752

Version: Final published version: final published article, as it appeared in a journal, conference proceedings, or other formally published context

Terms of Use: Article is made available in accordance with the publisher's policy and may be subject to US copyright law. Please refer to the publisher's site for terms of use. 


\title{
On the measurement of intensity correlations from laboratory and astronomical sources with SPADs and SNSPDs
}

\author{
Edward Schroeder ${ }^{\mathrm{a}}$, Philip Mauskopf ${ }^{\mathrm{ab}}$, Genady Pilyavsky ${ }^{\mathrm{b}}$, Adrian Sinclair ${ }^{\mathrm{a}}$, Nathan Smith $^{\mathrm{b}}$, \\ Sean Bryan ${ }^{\mathrm{b}}$, Hamdi Mani ${ }^{\mathrm{b}}$, Dmitry Morozov ${ }^{\mathrm{c}}$, Karl Berggren ${ }^{\mathrm{d}}$, Di Zhu ${ }^{\mathrm{d}}$, Konstantin \\ Smirnov ${ }^{\mathrm{e}}$, and Yuriy Vakhtomin ${ }^{\mathrm{e}}$ \\ ${ }^{a}$ Department of Physics, Arizona State University, Tempe, AZ, United States \\ ${ }^{\mathrm{b} S c h o o l}$ of Earth and Space Exploration, Arizona State University, Tempe, AZ, United States \\ ${ }^{\mathrm{c} S c h o o l ~ o f ~ P h y s i c s ~ a n d ~ A s t r o n o m y, ~ C a r d i f f ~ U n i v e r s i t y, ~ C a r d i f f, ~ W A L, ~ U n i t e d ~ K i n g d o m ~}$ \\ ${ }^{\mathrm{d}}$ Department of Electrical Engineering and Computer Science, Massachusetts Institute of \\ Technology, Cambridge, MA, United States \\ esuperconducting Nanotechnology (SCONTEL), Moscow, Russian Federation
}

\begin{abstract}
We describe the performance of detector modules containing silicon single photon avalanche photodiodes (SPADs) and superconducting nanowire single photon detectors (SNSPDs) to be used for intensity interferometry. The SPADs are mounted in fiber-coupled and free-space coupled packages. The SNSPDs are mounted in a small liquid helium cryostat coupled to single mode fiber optic cables which pass through a hermetic feed-through. The detectors are read out with microwave amplifiers and FPGA-based coincidence electronics. We present progress on measurements of intensity correlations from incoherent sources including gas-discharge lamps and stars with these detectors. From the measured laboratory performance of the correlation system, we estimate the sensitivity to intensity correlations from stars using commercial telescopes and larger existing research telescopes.
\end{abstract}

Keywords: single photon avalanche diode, superconducting nanowire single photon detector, Hanbury Brown and Twiss, intensity interferometry, time-to-digital converter, FPGA, ROACH2

\section{INTRODUCTION}

Stars are incoherent (thermal) sources of light that radiate over a wide range of frequencies. Correlations in the intensity of filtered thermal radiation can be measured within a coherence time that is determined by the central wavelength and bandwidth of the filtered light by correlating signals from two or more detectors observing the same source. Moreover, the amplitude of this second order correlation function is a function of spatial separation between detectors and depends on the angular size of the source. Measurements of diameters of stars using this technique, called intensity interferometry, were first accomplished by Hanbury Brown and Twiss (HBT) in $1956 .{ }^{1}$ In this paper, we describe initial results from measurements of intensity correlations from laboratory sources and the experimental setup for measuring diameters of bright stars with small to moderate sized telescopes using silicon-based single photon avalanche diodes (SPADs) and superconducting nanowire single photon detectors (SNSPDs).

\footnotetext{
Further author information:

Genady Pilyavsky (General correspondence): gcp5017@gmail.com

Edward Schroeder (Superconducting detectors): edward.schroeder@asu.edu

Adrian Sinclair (FPGA-based TDC design): adrian.sinclair@asu.edu
} 


\section{INTENSITY INTERFEROMETRY}

\subsection{THE CORRELATION FUNCTION}

The correlation function is an nth-order function that relates the electromagnetic field at multiple spacetime points. The first-order correlation function is given by

$$
C_{(1)}\left(r_{1} t_{1}, r_{2} t_{2}\right)=\left\langle E^{*}\left(r_{1} t_{1}\right) E\left(r_{2} t_{2}\right)\right\rangle,
$$

where $E$ is the electric field and $r t$ represents the position in space and time of the detectors. For $r_{1}=r_{2}$ and $t_{1}=t_{2}, C_{(1)}$ is simply the intensity of the field. The second-order correlation function is measured by correlating the intensity of the radiation field emitted by a source at two different detectors separated in space and time. Coherent radiation exhibits random intensity fluctuations due to the stochastic nature of the arrival and detection time of the photons that are uncorrelated between any two detectors measuring the same source. However, incoherent light exhibits additional intensity fluctuations corresponding to photon "bunching" that are correlated at some level between detectors.

The second-order correlation function is given $\mathrm{as}^{2}$

$$
C_{(2)}\left(r_{1} t_{1}, r_{2} t_{2}\right)=\left\langle I\left(r_{1} t_{1}\right) I\left(r_{2} t_{2}\right)\right\rangle,
$$

where $I$ is the intensity of the electric field at the detectors. For $r_{1} \neq r_{2}$ and $t_{1}=t_{2}, C_{(2)}$ provides a spatiallydependent measurement of the wavefront state.

The signal-to-noise ratio for detecting second order intensity fluctuations from an incoherent light source is given by

$$
\frac{S}{N}=\epsilon n_{o c c} N_{\text {modes }} \sqrt{\frac{\tau_{\text {int }}}{\tau_{\text {det }}}} C_{(2)}\left(r_{1} t_{1}, r_{2} t_{2}\right),
$$

where $\epsilon$ is the overall efficiency of the system, $n_{\text {occ }}$ is the occupation number of a single radiation mode, $N_{\text {modes }}$ is the number of expected modes, $\tau_{i n t}$ is the integration time, $\tau_{\text {det }}$ is the detector time constant, and $C_{(2)}\left(r_{1} t_{1}, r_{2} t_{2}\right)$ is the normalized second-order correlation function as discussed previously (see A.2 for the derivation of signalto-noise).

$C_{(2)}\left(r_{1} t_{1}, r_{2} t_{2}\right)$ is experimentally obtained by varying the distance between the two detectors. The maximum separation between detectors or baseline where spatial correlations can be detected is given approximately by the Rayleigh criterion:

$$
\sin \theta=\frac{1.22 \lambda}{d}
$$

where $\theta$ is the angular size of the source, $\lambda$ is the central wavelength of the observed bandwidth of light, and $d$ is the baseline.

The baselines required to determine stellar diameters are on the order of a kilometer. ${ }^{3}$ Intensity interferometry has advantages for large baselines compared to conventional phase interferometry. Phase interferometry requires the light from multiple telescopes observing the same source to be physically combined so that an interference pattern is formed and can be analyzed while maintaining phase coherence. Fluctuations in the path lengths between telescopes and through the atmosphere limit the duration of a fixed fringe pattern and therefore the detectable source magnitude.

For intensity interferometry, instead of the physical recombination of the beams, the time streams from two detectors corresponding to the arrival times of photons can be stored digitally. In post-processing, the two steams can be combined and correlations can be determined. Therefore, in principle any baseline can be used for intensity interferometry measurements.

By increasing the distances over which correlated beams of light can be analyzed, incoherent light sources with small angular diameters can be measured. In addition to typical stellar objects such as Sirius, other extra-solar objects may be considered for measurement such as neutron stars and perhaps even exoplanets. Since there is no

loss in signal by adding additional baselines in intensity interferometry, a large array of commercially available telescopes with a combined area equal to or greater than existing large telescopes may be used. 


\section{INSTRUMENTATION}

\subsection{IMPORTANT PARAMETERS}

The experimental parameters of a detector system for intensity interferometry consist of: i) detection efficiency, ii) system dead time (and afterpulsing probability), iii) maximum count rate, iv) dark count rate and v) detector jitter. The detection efficiency of commercial SPADs is apprximately $50 \%$. In the next section, we describe the measurement of our system efficiency and the efficiency of the SNSPDs. The system dead time is the amount of time it takes for a detector to return to its original state after a detetction occurs. The SPADs have pulse widths of 10-20 ns and a dead time of 50-100 ns. The SNSPDs have pulse recovery times of 5-10 ns. We use a variable system dead time from 50-100 ns in the readout electronics to reduce the effects of afterpulsing in the SPADs. The maximum count rate for the SPADs was 2-10 MHz and for the SNSPDs was > $10 \mathrm{MHz}$. The dark count rates for all detectors were on order or less than $1 \mathrm{kHz}$ and therefore insignificant compared to the typical photon detection rates. Jitter is the uncertainty in the arrival time of an individual photon at a detector. For the SPADs the typical jitter is 350-600 ps while the SNSPDs have jitters of 10s of ps.

\subsection{SPAD OPERATION}

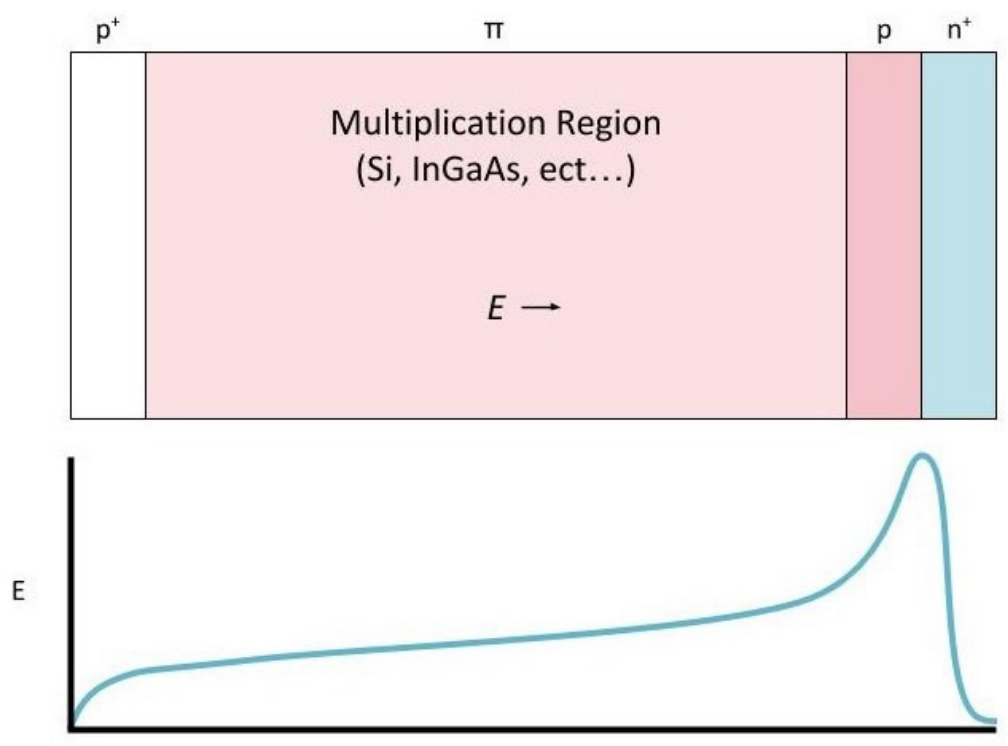

Figure 1. The interior of a SPAD showing how the electric field across the length of the multiplication region induces the avalanche effect.

SPADs operate under a voltage bias just above the breakdown voltage. ${ }^{4}$ A single photon event causes the semiconductor material to conduct and commences the avalanche effect. See Figure 1 to see how the electric field varies in the detector. When a photon creates an electron-hole pair in the multiplication region of a SPAD, the strong E-field formed at the p-n junction motivates impact ionization, resulting in an avalanche pulse that coincides with a photon arrival within hundreds of picoseconds of time jitter. The SPAD returns to an operational state by resetting the voltage bias.

\subsection{SNSPD OPERATION}

SNSPDs are capable of detecting single photons with much less timing jitter than SPADs and can enable an improved signal-to-noise ratio in the HBT experiment. SNSPDs have a typical timing jitter of less than $100 \mathrm{ps},{ }^{5}$ while SPADs have a typical timing jitter of less than or equal to 600 ps. SNSPDs produce a voltage pulse when a single incident photon strikes the superconducting nanowire, or active area, of the detector. The nanowires we used are made of $\sim 4 \mathrm{~nm}$ niobium nitride $(\mathrm{NbN})$ films. They have a typical width of $<100 \mathrm{~nm}$, and are meandered to cover an area of approximately $100 \mu \mathrm{m}^{2}$. 


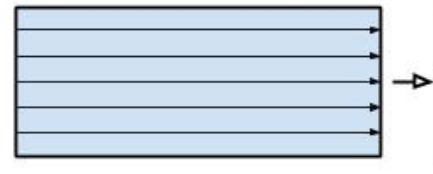

(a)

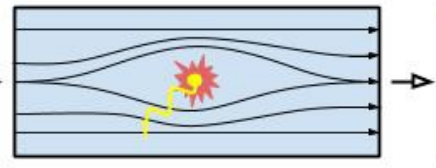

(b)

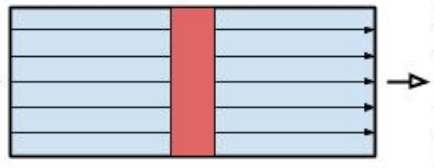

(c)

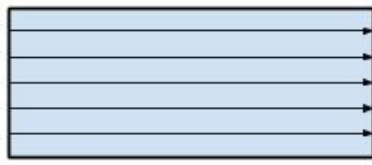

(d)

Figure 2. Detection mechanism of an SNSPD. (a) The superconducting state of a section of nanowire biased with a current below the switching current. (b) When a photon is absorbed by the nanowire, it creates a hotspot. The current is repelled to the sides of the nanowire. (c) The critical current density is exceeded and a resistive strip is formed across the width of the nanowire, shunting the current and resulting in a readable voltage pulse. (d) The bias current is diverted to the load, and resistive region is dissipated. The nanowire returns to the superconducting state.

When cooled below the critical temperature of $\mathrm{NbN}$, the nanowire becomes superconducting. When the superconducting nanowire is biased close to its switching current, a single photon carries enough energy to drive a section of the nanowire normal. ${ }^{6}$ Initially, the superconducting state of a section of nanowire is undisturbed with a bias current just below the critical current. At some point, a photon strikes this section of the wire. This incident photon is absorbed and a resistive hotspot is formed. The current is repelled to the sides, causing the local current density to exceed the critical current density. The hotspot then grows and switches the entire cross section of the nanowire to the resistive state. The current is completely blocked such that a measurable voltage pulse is generated. Finally, since the bias current is diverted to the load, there is not enough current in the nanowire to sustain the hotspot, and the nanowire returns to the superconducting state.

\subsection{SNSPD PACKAGING}
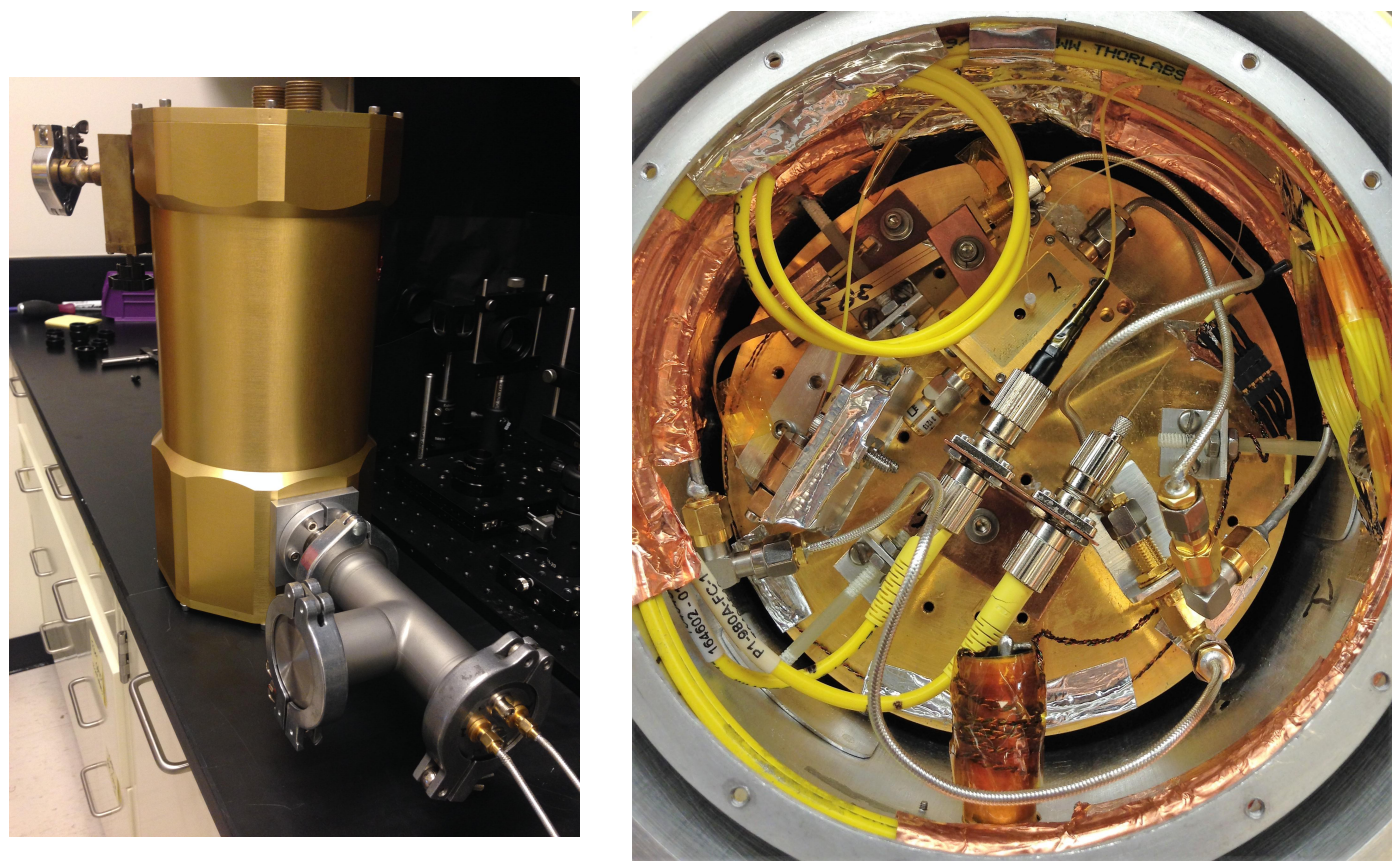

Figure 3. Left: The Infrared Labs, Inc. dewar. Right: The set-up inside of the dewar.

Two SNSPDs from different collaborators were used in this experiment. The first SNSPD was obtained from Cardiff University and was fabricated by SCONTEL. This device was characterized at ASU where we determined the critical temperature of the device to be $9.65 \mathrm{~K}$. This device was pre-packaged and fiber-coupled. The second device was fabricated at the Massachusetts Institute of Technology. This device was packaged and coupled to 
fiber optics at ASU. The package consisted of a chip mount with a lid that contained holes for alignment pins and fiber optic ferrules. We aligned the fiber to the nanowires on the chip using an optical microscope and we estimated our alignment accuracy to be approximately $30 \mu \mathrm{m}$. Both detectors were mounted in a small Infrared Laboratories, Inc. dewar (see Figure 3).

Based on the uncertainty in alignment, we calculated the expected efficiency as a function of vertical separation between the fiber and the nanowire. Given the numerical aperture of our single-mode fiber $(\mathrm{NA}=0.13)$, a vertical distance of $300 \mu \mathrm{m}$ between the ferrule and the detector chip yields a spot size of diameter $78.7 \mu \mathrm{m}$ compared to the detector diameter of $15.0 \mu \mathrm{m}$. Therefore we expected the coupling efficiency to be only a few percent (see Appendix A.1).

The fiber used to couple to the MIT detector was made in-house with materials from ThorLabs, Inc. On the detector side, a bare ferrule was inserted with single-mode fiber and polished. The other side was fitted with a FC-connector in order to couple with a FC-FC connector to a matching single-mode fiber through a hermetic feed-through also made at ASU. This feedthrough contains two SMA input/output ports that lead to the two detectors.

\section{LAB SET-UP}

\subsection{OPTICAL SET-UP}

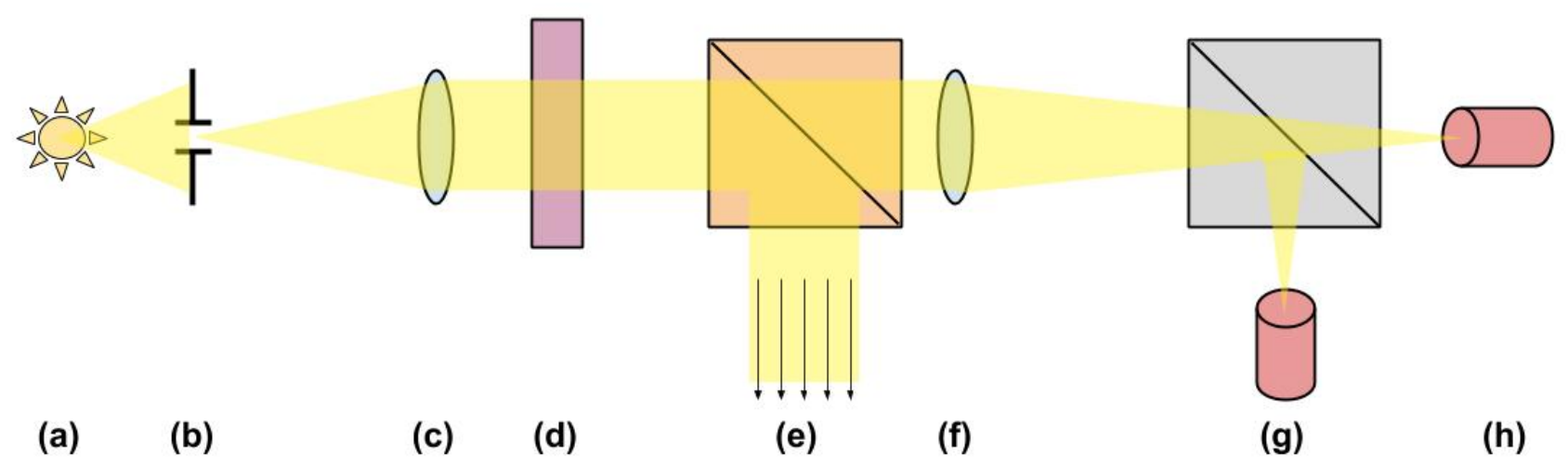

Figure 4. (a) Thermal light source (b) $12.5 \mu \mathrm{m}$ pinhole (c) $2.5 \mathrm{~cm}$ lens (d) Narrow band filter (e) Polarizing beam splitter (f) $2.5 \mathrm{~cm}$ lens (g) Beam splitter and detector channel 1 (h) Detector channel 2

An optical table set-up was fashioned to allow us to detect spatial intensity correlations with a laboratory thermal source. Our artificial star consists of a $12.5 \mu \mathrm{m}$ pinhole illuminated by a $\simeq 2100 \mathrm{~K}$ incandescent lamp. A $2.5 \mathrm{~cm}$ diameter lens is placed at its focal length of $\sim 40 \mathrm{~cm}$ from the pinhole in order to collimate the light which is then passed through a narrow band filter. Collimation of the light beam is necessary for efficient operation of the filter. From here, the collimated beam is fed through a 50/50 polarizer as differing polarizations are uncorrelated. Now that the beam has some bandwidth $\Delta \nu$ and is polarized, the light is passed through an additional $2.5 \mathrm{~cm}$ lens with a $\sim 30 \mathrm{~cm}$ focal length for refocusing into the two detectors via a $50 / 50$ beam splitter.

For the SPAD set-up, the light for one channel is free-space coupled and the light for the other channel is fiber-coupled. The active area in both instances is $200 \mu \mathrm{m}$ in diameter, which makes alignment with xyzmicrometer stages straightforward as the size of the beam is on the order of $10 \mu \mathrm{m}$. For the SNSPD set-up, the beam is fed into two single-mode fibers with an active area of $10 \mu \mathrm{m}$, which makes alignment more difficult.

\subsection{READOUT}

The electronic pulses from both detectors must be amplified before entering the readout electronics. The commercial SPADs have integrated pulse shaping and provide a TTL $5 \mathrm{~V}$ or LVCMOS $3.3 \mathrm{~V}$ signal which may be easily interfaced. The SNSPDs require a relatively high frequency microwave readout chain with low phase 
noise (timing jitter) components. This microwave readout chain consists of a low noise current source which is low-pass filtered to the DC port of a Mini-Circuits BIAS-TEE (ZFBT-4R2G+). The AC+DC port biases the nanowires and the AC output port is amplified with two Mini-Circuits amplifiers (ZKL-1R5+). To reduce the risk of standing waves forming in the AC output chain, an attenuator is added before and after the first amplifier. This chain is successful in producing $1.5 \mathrm{~V}$ pulses which are capable of being read by the timing electronics.

\subsection{FPGA-based TDC}

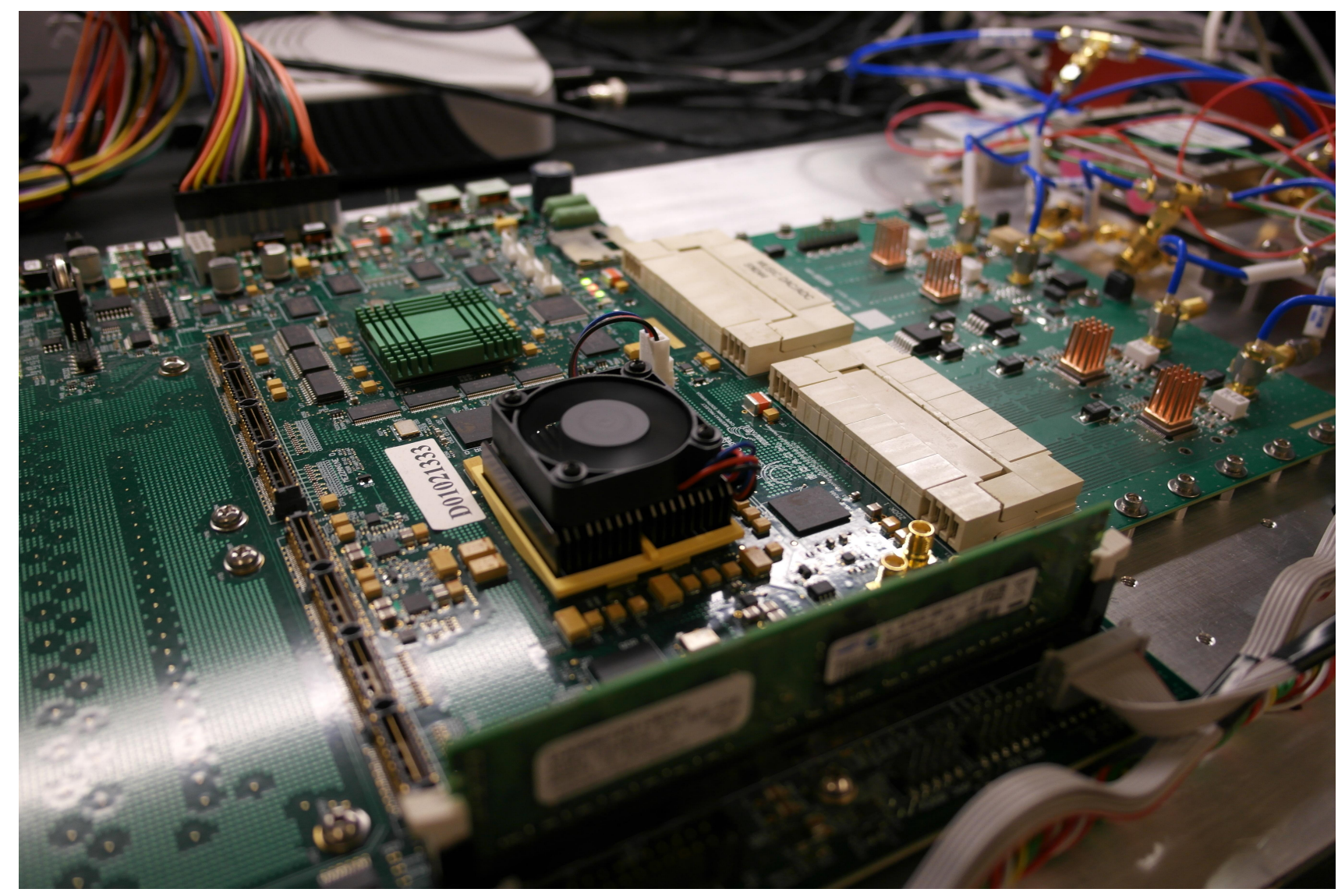

Figure 5. A picture of the ROACH2 board equipped with a custom TDC that was configured and programmed at ASU to be capable of preforming A to B interval measurements.

To read out both types of detectors, a time-to-digital converter (TDC) is used. Our intention is to use TDCs that are configured to measure the time interval between two electronic pulses which start on one channel $\mathrm{A}$ and end on another channel $\mathrm{B}(\mathrm{A} \rightarrow \mathrm{B})$. The correlations will be stored in these time differences.

In our investigation of commercial TDCs we discovered that while many have timing resolutions in the tens of picoseconds range, the data throughput rate was unable to keep up with the photon count rates desired..$^{7,8}$ Meanwhile the development of an in-house TDC implemented on a field programmable gate array (FPGA) has been underway. This has allowed for the custom tailoring of the instrument to the experimental requirements such as continuous measurements and data streaming. The current design has a resolution of $4 \mathrm{~ns}$ and a tested input count rate of $10 \mathrm{MHz}$ with a sustainable continuous readout. The collaboration for astronomy signal processing and electronics research $\left(\mathrm{CASPER}^{9}\right)$ has allowed for the rapid development of this instrument using their re-configurable open architecture computing hardware (ROACH2, ${ }^{10}$ Figure 5).

The current TDC design consists of three main parts: a coarse counter, phase measurement, and a read/write output buffer. The coarse counter is a digital counter which increments by one unit with each successive clock 


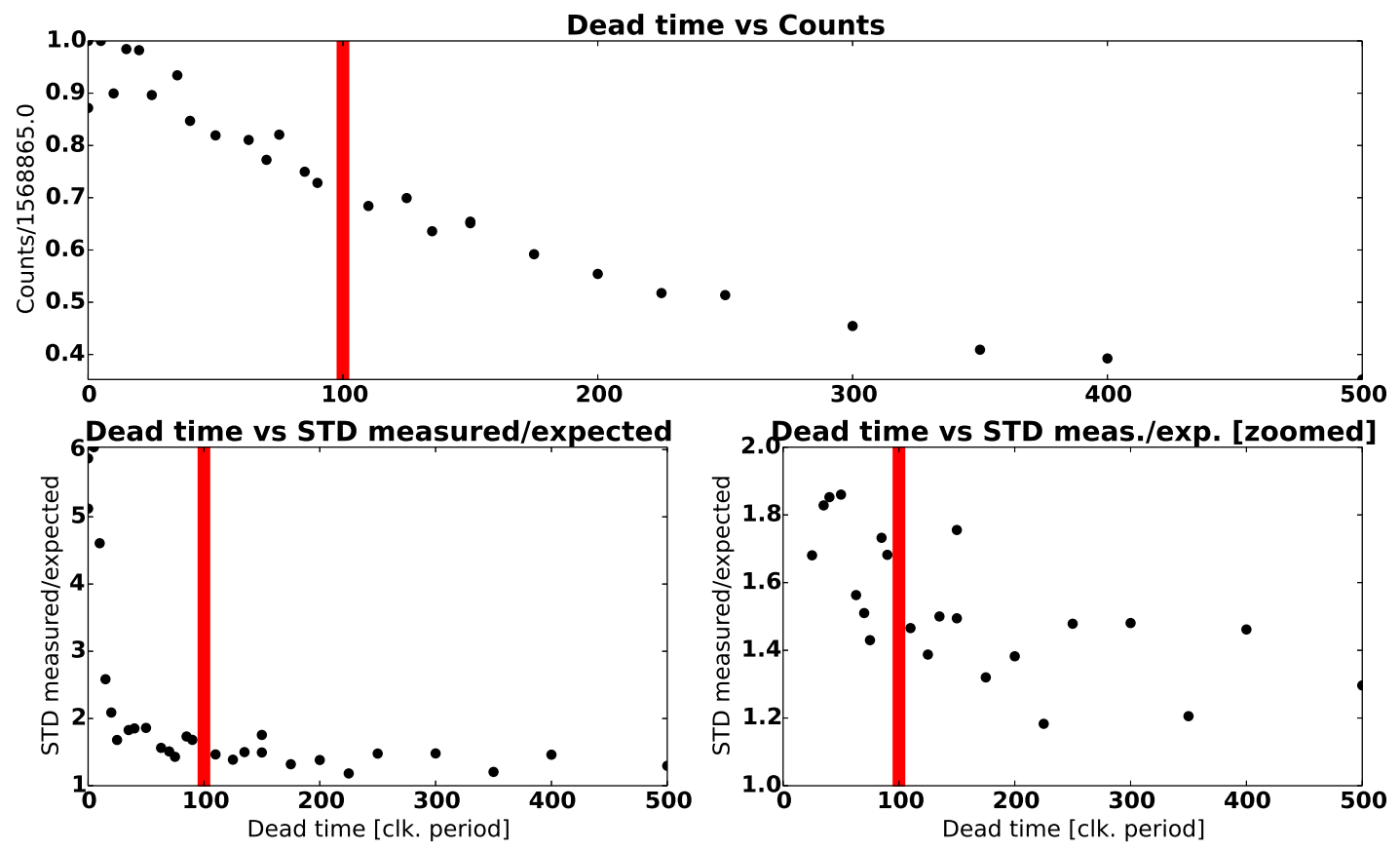

Figure 6. Top: Number of coincident counts as a function of programmable dead time. Each bin is one clock period (4 ns). Bottom Left: Measured standard deviation in coincident counts normalized by the expected standard deviation. Bottom Right: Zoomed in version of the plot on the left. The red line is the selected dead time.

cycle. The phase measurement is a measure of the rising edges of the pulses with respect to the clock phase. The combination of the coarse count and two phase measurements gives the time interval in units of fractional clock cycles. Finally this measurement is read out using a read/write output buffer.

To test the TDC's histogram bin widths, a code density test was performed using a SPAD as a random event generator. A programmable deadtime for each channel was added to the design to reduce the risk of false events due to reflections or after pulsing. Figure 6 presents some of the results of these tests where it is shown that the standard deviation of the resulting distribution is dependent upon the deadtime. Areas of future development include increasing the number of channels, increasing the timing resolution using delay lines, on-board error correction, and the synchronization of TDCs across multiple ROACH2 boards.

\section{LAB RESULTS}

Two main results from the laboratory tests were i) measurement of the system efficiency for the different detectors and ii) characterization of the instrument baseline signal and confirmation that the noise integrates down over a long intergration time.

\subsection{SPAD EFFICIENCY}

We have two models of SPADs that we are using in our intensity interferometry set-up. The first one is a 4-channel SPAD from Excelitas, module SPCM-AQ4C. Each channel has a maximum count rate of $2 \mathrm{MHz}$ with a $600 \mathrm{ps}$ timing resolution. The typical peak quantum efficiency of this detector is $60 \%$ at $650 \mathrm{~nm}$. We only use one channel in this module due to unavoidable cross-talk between channels and therefore significant noise that washes out any chance of measuring intensity fluctuations.

The second model that we are using is a single channel SPAD from Excelitas, module SPCM-AQRH. The maximum count rate is about $15 \mathrm{MHz}$ with a $350 \mathrm{ps}$ timing resolution. The typical peak quantum efficiency 
of this detector is $70 \%$ at $700 \mathrm{~nm}$. With these detectors, we measured a count rate at a wavelength of $671 \mathrm{~nm}$ with $1 \mathrm{~nm}$ bandpass filter of $\sim 7 \mathrm{MHz}$. This is consistent with the expected detector efficiency combined with the polarization splitter and beam divider assuming an effective source temperature of $2100 \mathrm{~K}$.

\subsection{SNSPD EFFICIENCY}

We also measured the efficiency for the set-up with the SCONTEL SNSPD compared to the SPAD. The SPCMAQ4C was used in an optical set-up that contained a $1000 \mathrm{~nm}$ filter with a $10 \mathrm{~nm}$ bandwidth and a single mode fiber. With this illumination the photon count rate in the SPAD was approximately $50 \mathrm{kHz}$. The same set-up was used and coupled to the SCONTEL SNSPD. For the same optical set-up, the photon count rate in the SNSPD was approximately $8.6 \mathrm{kHz}$. The number of detected photons can be expressed by

$$
N=\gamma \epsilon_{S P A D}
$$

where $\gamma$ is the number of photons incident on the detector and $\epsilon_{S P A D}$ is the efficiency of the SPAD.

Given that the efficiency of the SPAD at $1000 \mathrm{~nm}$ is approximately $10 \%{ }^{11}$ the efficiency of the SNSPD is then found to be $\epsilon_{S N S P D}=1.7 \%$ at $1000 \mathrm{~nm}$.

\subsection{INSTRUMENTAL NOISE REMOVAL}

We collected data using the set-up described in Figure 4. The coincident photon distribution was dominated by instrumental noise, which was well above the expected Poisson noise level. While the exact source of the instrumental noise has yet to be determined, we attempted to remove it by collecting baseline data where no correlation signal was expected to be observed. This was done by removing the filter and lowering the brightness of the source to match the photon count rate obtained with the filter. In addition, we removed the throughput limiting aperture, introducing multiple modes, and placed the detectors at different locations within the beam. Lastly, the cables connected to the SPADs were reversed so that the bin in the histogram where an excess coincident signal was expected was shifted to be outside of the range of the histogram.

We subtracted the two data sets and the standard deviation of the result was closely matched to the expected Poisson noise (Figure 8). Although additional tests are required, the preliminary results show that the instrumental noise can be successfully removed. Furthermore, the baseline measurement can be done on the FPGA itself, concurrently with the data collection. The output can be the $\mathrm{A} \rightarrow \mathrm{B}$ measurements, as well as $\mathrm{B} \rightarrow \mathrm{A}$, created with an internal FPGA delay line.

\subsection{EXPECTED SIGNAL-TO-NOISE}

The expected signal-to-noise ratio for our zero-point correlation is given by

$$
\frac{S}{N}=\epsilon n_{o c c} N_{\text {modes }} \sqrt{\frac{\tau_{\text {int }}}{\tau_{\text {det }}}},
$$

where $\epsilon$ is the overall efficiency of the system, $n_{o c c}$ is the occupation number of a single radiation mode, $N_{\text {modes }}$ is the number of expected modes, $\tau_{\text {int }}$ is the integration time, and $\tau_{\text {det }}$ is the detector time constant. Note that $\Gamma^{2}(d, \nu)=1$ for zero-point measurements and therefore is not included here.

In our SPAD-based experiment, a $671 \mathrm{~nm}$ filter with a bandwidth of $1 \mathrm{~nm}$ is used. The temperature of the source is $2100 \mathrm{~K}$. The pinhole is placed $\sim 40 \mathrm{~cm}$ from the collecting lens such that $N_{\text {modes }} \leq 1$, and the collimated light is passed through the filter/polarizer. The beam is then refocused, passed through a 50/50 beam slitter, and falls onto the fibers. A photon count rate of $\sim 7 \mathrm{MHz}$ in both channels has been observed, suggesting that the overall system efficiency is $\sim 24 \%$. In a $\sim 20$ hour integration, with the timing resolution of our current custom TDC $\left(\tau_{d e t}=\tau_{t d c}=4 \mathrm{~ns}\right)$, we expect a $\frac{S}{N}$ of $\sim 23$. Future TDC development is expected to increase the timing resolution to $312 \mathrm{ps}\left(\tau_{t d c}\right)$, thereby making the system detector limited $\left(\sim 500 \mathrm{ps}, \tau_{d e t}\right)$ and decreasing the required integration time by a factor of 13 in order to obtain the same $\frac{S}{N}$ of 23 in just $\sim 3$ hours.

We are operating a similar experiment with the SNSPDs carried out at $1150 \mathrm{~nm}$ (bandwidth of $10 \mathrm{~nm}$ ) at which the detectors are most sensitive to incident photons. This setup has three advantages over the previously 

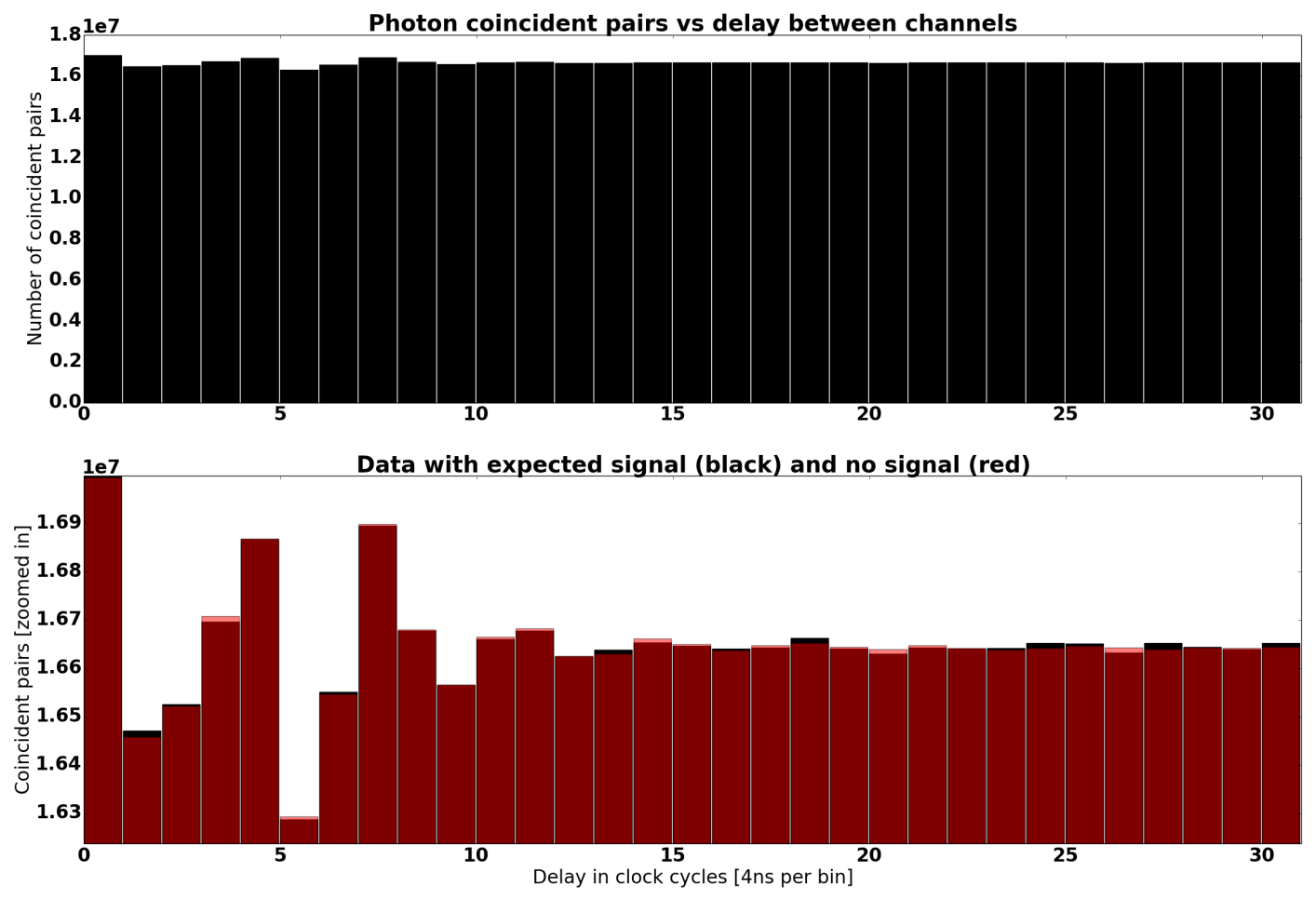

Figure 7. Top: Histogram displaying the number of coincident photons as a function of time. Each bin represents 1 clock period of 4 ns. Bottom: Zoomed in version of the top plot. The red bins represent data which should, in principal, contain correlated photons. The black represents counts which were obtained from an uncorrelated light source.

described one that utilizes SPADs. Observations carried out at $\sim 2 \times$ longer wavelengths allows us to place the collimating lens at half the distance $(20 \mathrm{~cm})$ which would increase the count rate by a factor of 4 while the source remains unresolved ( $\leq 1$ mode). Additionally, the observational wavelength is closer to the peak of the black body radiation thereby increasing the occupation number and thus the photon rate due to the combination of higher energy output from the source and lower photon energy. Finally, the timing resolution of the detectors $(\sim 50 \mathrm{ps})$ is superior compared to that of the TDC, meaning $\tau_{d e t}=\tau_{t d c}=312 \mathrm{ps}$. The photon count-rate will equal to that of the SPAD setup at $671 \mathrm{~nm}$ (bandwidth of $1 \mathrm{~nm}$ ) for a system efficiency that is $\sim 0.06 \%$, which gives sufficient margin in the fiber-to-SNSPD coupling while still obtaining $\frac{S}{N}$ of $\sim 23$ in $\sim 20$ hours.

\section{PROSPECTS FOR ASTRONOMY}

\subsection{EXISTING OPTICAL TELESCOPES}

A key motivation for this experiment is the pre-existence of suitable telescopes. Commercially available telescopes in the 8-20 inch range can be used. In addition to convenience, this is useful because the more telescopes that are integrated into the system, the more baselines there are to construct. The signal-to-noise scales as the square root of the number of baselines.

Medium and large optical telescopes arrays are also available to the scientific community. For example, Kitt Peak National Observatory in Arizona has many suitable telescopes that can provide baselines up to 650 meters. ${ }^{12}$ 


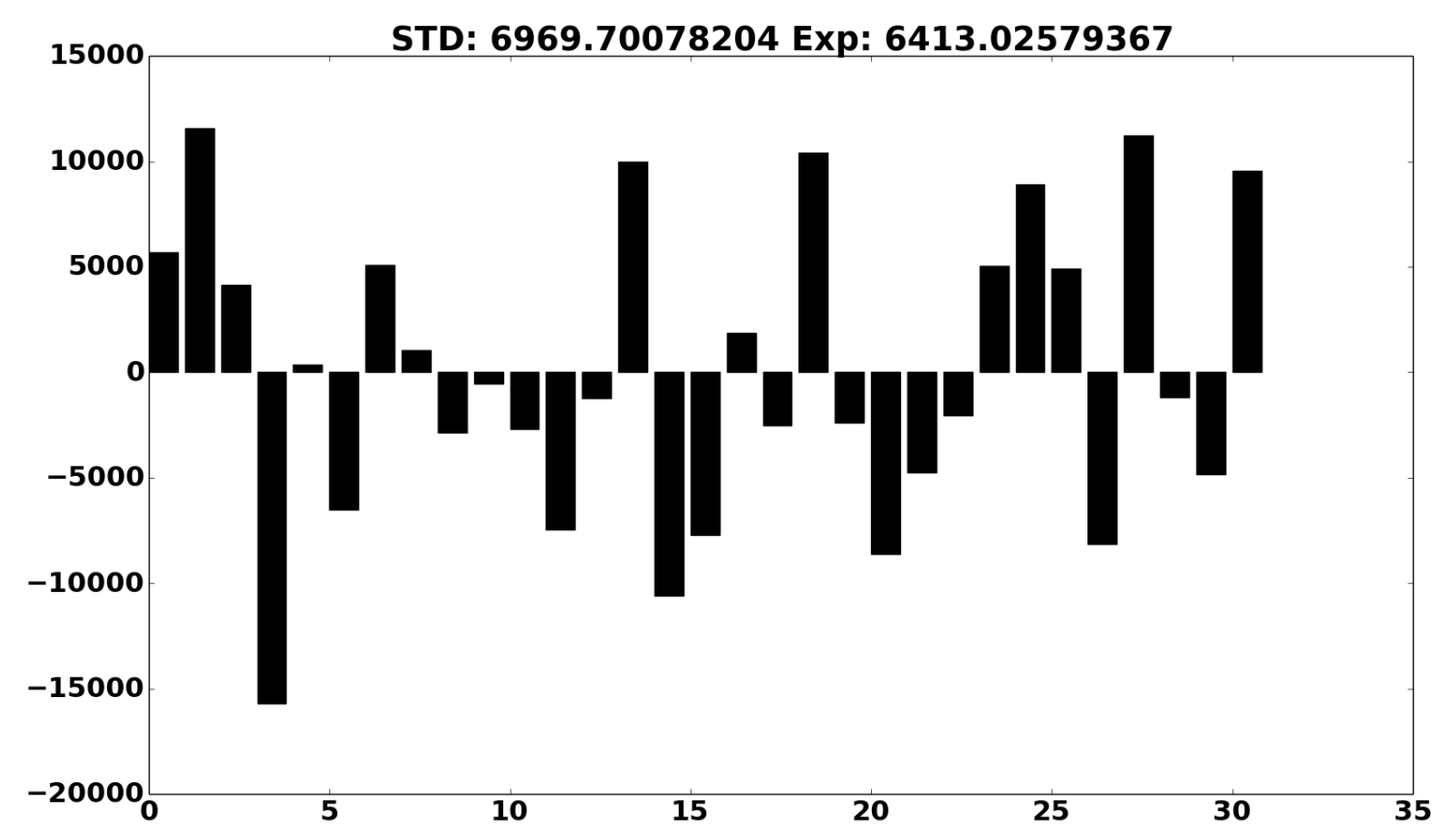

Figure 8. The difference in count rate form the data set expected to have correlations and the data set with no correlations as a function of time (Each bin is $4 \mathrm{~ns}$ ). The measured standard deviation is close to the Poisson deviation expected in the absence of a signal.

\subsection{MAGNITUTE LIMITS ACHIEVABLE}

The number of photons per second per Hertz from a selected source of magnitude $M$ can be found via the following equation:

$$
\Gamma_{\gamma}=0.05 \lambda f_{o} 2.512^{\Delta m} A
$$

where $\lambda$ is the observed central wavelength, $f_{o}$ is the calibrated flux (in Jy) at a given $\lambda$ of an $m=0$ star, ${ }^{13-15}$ $\Delta m$ is the change in magnitude of the star from $m=0$, and $A$ is the collecting area of the telescope.

The signal-to-noise ratio is then related to the magnitude of the target by

$$
\frac{S}{N}=\epsilon 0.05 \lambda f_{o} 2.512^{\Delta m} A \sqrt{\frac{\tau_{\text {int }}}{\tau_{\text {det }}}},
$$

Table 6.2 shows the achievable magnitudes based on a zero-point measurement with $\frac{S}{N}=10$ and for different values of the parameters $\lambda$, telescope diameter $d_{\text {tele }}$, and $\tau_{\text {det }} . \tau_{\text {det }}$ is taken to be the achievable timing resolutions from SPADs and SNSPDs. $\tau_{\text {int }}$ was taken to be the course of a single night, or 8 hours. An overall system efficiency of $5 \%$ was assumed.

\section{CONCLUSION}

A proof-of-concept intensity interferometry experiment is being realized at ASU. To obtain zero-point correlations with SNSPDs in the lab and on the sky, the overall efficiency of the coupling must be improved. To optimize the efficiency, we will improve the fiber alignment onto the SNSPD detectors and improve the stability and alignment of the portable optical bench in our system. Additionally, the signal-to-noise of the measurement will be improved by increasing the timing resolution and number of channels available on the the FPGA-based TDC. 
Table 1. Limiting magnitude of stars for which zero-point correlation is achievable in 8 hours with $5 \%$ system efficiency. The calibration flux $\left(f_{o}\right)$ was obtained from the Gemini Observatory magnitudes-to-flux calculator (link).

\begin{tabular}{ccccc}
$\begin{array}{c}\text { Observation } \\
\begin{array}{c}\text { Wavelength } \\
(\mathrm{nm})\end{array}\end{array}$ & $\begin{array}{c}\text { Calibration } \\
\text { Flux } \\
(\mathrm{Jy})\end{array}$ & $\begin{array}{c}\text { Telescope } \\
\text { Diameter } \\
(\mathrm{m})\end{array}$ & $\begin{array}{c}\text { Detector } \\
\text { Resolution } \\
(\mathrm{ps})\end{array}$ & $\begin{array}{c}\text { Apparent } \\
\text { Magnitude } \\
\text { Limit }\end{array}$ \\
\hline & & & & \\
671 & 2841 & 0.36 & 500 & Sirius $\left(m_{R}\right)$ \\
671 & 2841 & 4.0 & 500 & $4.1\left(m_{R}\right)$ \\
1150 & 1725 & 0.36 & 60 & $0.1\left(m_{J}\right)$ \\
1150 & 1725 & 4.0 & 60 & $5.3\left(m_{J}\right)$ \\
\hline
\end{tabular}

Further improvement of the signal-to-noise will come from the characterization of the instrumental noise and the possibility of mitigating it or removing it altogether.

Once the system is optimized and intensity correlations are measured in the lab, the optical set-up will be coupled to telescopes and a stellar diameter will be measured. When this is accomplished, intensity interferometry will have been reinstated as a viable science tool with commercial single photon detector technology.

\section{APPENDIX A. CALCULATIONS}

\section{A.1 PERCENTAGE OF LIGHT FALLING ON A DETECTOR}

The percentage of light falling on a detector can be approximated by

$$
\%=\left(\frac{d_{\text {snspd }}}{d_{\text {spot }}}\right)^{2}=\left(\frac{d_{\text {snspd }}}{2 * z * \tan (\arcsin (N A))}\right)^{2},
$$

where $d_{\text {snspd }}$ is the diameter of the detector, $d_{\text {spot }}$ is the diameter of the spot of light incident on the detector chip, $z$ is the ferrule-to-chip vertical displacement, and $N A$ is the numerical aperture of the fiber optic cable.

Plugging in $z=300 \mu m$ and $N A=0.13$, we get

$$
\%=\left(\frac{15 \mu m}{2 * 300 \mu m * \tan (\arcsin (0.13))}\right)^{2}=2.63
$$

\section{A.2 SIGNAL-TO-NOISE DERIVATION}

Our sources are incoherent and therefore follow Bose-Einstein statistics. The occupation number in this case is

$$
n_{\text {occ }}=\frac{1}{e^{\frac{h \nu}{k T}}-1},
$$

where $h$ is Plank's constant, $\nu$ is the central frequency of the light, $k$ is Boltzmann's constant, and $T$ is the temperature of the source.

The occupation number is the number of photons per second per mode per Hertz and is representative of the probability that a photon is occupying a particular state. If two detectors are measuring emitted radiation from a single source, then the probability that both detectors will measure a photon in the same state (i.e. the same time) is $P=n_{o c c}^{2}$. If we integrate for some amount of time $\tau_{i n t}$, we will detect the following number of photons:

$$
n_{\text {correlations }}=\epsilon \Gamma_{\text {det }} n_{\text {occ }} \tau_{\text {int }},
$$

where $\Gamma_{\text {det }}$ is the number of photons per second detected by a detector, $n_{\text {occ }}$ is the occupation number, and $\tau_{\text {int }}$ is the integration time. These are the correlated photons (i.e. the signal). Multiplying $\Gamma_{\text {det }}$ and $\tau_{\text {int }}$ gives the number of photons counted at one detector in a given amount of time and multiplying this value by the 
probability of detecting a photon in the same state at the second detector gives the number of correlations. This can be written as

$$
S=\epsilon^{2} n_{o c c}^{2} \Delta \nu \tau_{i n t} .
$$

In addition to photons that are occupying the same state causing these detected coincidences, there are random coincidences that are not correlated. This is where the noise associated with this measurement arises. Consider two detectors A and B. The number of coincidences that are random can be determined by multiplying the number of coincidences in A by the probability that for a particular photon detected in $\mathrm{A}$, a photon is detected in $\mathrm{B}$ within the detector time constant $\tau_{\text {det }}$.

The number of coincidences in $\mathrm{A}$ is given by

$$
n_{A}=\epsilon n_{o c c} \Delta \nu \tau_{i n t} .
$$

The probability that another photon will be detected in B within $\tau_{\text {det }}$ is

$$
P(B: A)=\epsilon n_{\text {occ }} \Delta \nu \tau_{\text {det }},
$$

where this is just the rate of photons detected by $\mathrm{B}$ in the time window for the photons to be considered coincident with a photon arriving at $\mathrm{A}$.

The number of random coincidences detected is then given by

$$
n_{\text {random }}=n_{A} P(B: A)=\epsilon^{2} n_{\text {occ }}^{2} \Delta \nu^{2} \tau_{\text {int }} \tau_{\text {det }} .
$$

Since the noise associated with random coincidences is Poissonian, we get

$$
N=\sqrt{n_{\text {random }}}=\epsilon n_{\text {occ }} \Delta \nu \sqrt{\tau_{\text {int }} \tau_{\text {det }}} .
$$

We arrive at the signal-to-noise ratio for the HBT experiment:

$$
\frac{S}{N}=\epsilon n_{\text {occ }} N_{\text {modes }} \sqrt{\frac{\tau_{\text {int }}}{\tau_{\text {det }}}} .
$$

\section{A.3 SYSTEM EFFICIENCY}

The efficiency of each detector channel can be determined by calculating the expected throughput of the system with a given configuration and comparing it to the measured count rates at each detector. In our system, the number of photons incident on a detector that are detected is given by

$$
\Gamma_{\text {det }}=\epsilon n_{\text {occ }} \Delta \nu N_{\text {modes }},
$$

where $\epsilon$ is the overall efficiency of the system leading up to a particular detector that determines the number of photons that are counted based on the number of photons that arrive at the beginning of the system, $n_{o c c}$ is the occupation number, $\Delta \nu$ is the observed bandwidth of the radiation field, and $N_{\text {modes }}$ is the number of modes.

The system efficiency for a given detector is then given by

$$
\epsilon=\frac{\Gamma_{d e t}}{\Gamma_{d e t}(\epsilon=1)},
$$

where $\Gamma_{d e t}$ is the measured count rate at the detector and $\Gamma_{d e t}(\epsilon=1)$ is the expected throughput given $100 \%$ efficiency.

The number of modes can be determined by comparing the minimum distance requirement for a single mode from the source to pass through the pinhole to the actual position of our pinhole. For the pinhole to be the size of one spatial mode, the resolving power needs to equal the angular size of the pinhole. In one-dimension, this is given by

$$
\frac{1.22 \lambda}{D_{\text {pinhole }}}=\frac{R_{\text {source }}}{d},
$$

where $\lambda$ is the central wavelength, $D_{\text {pinhole }}$ is the pinhole diameter, $R_{\text {source }}$ is the source radius, and $d$ is the distance from the source to the pinhole. For $\lambda=650 \mathrm{~nm}, d=0.2 \mathrm{~m}$. This means that as long as the pinhole is 0.2 $\mathrm{m}$ or greater from the source, only light from a single spatial mode is incident on our detectors. For zero-point correlation measurements, this is ideal. 


\section{REFERENCES}

[1] Hanbury Brown, R., "A Test of a New Type of Stellar Interferometer on Sirius," Nature 178, 1046-1048 (Nov. 1956).

[2] Foellmi, C., "Intensity interferometry and the second-order correlation function $\mathrm{g}^{(2)}$ in astrophysics," Astronomy and Astrophysics 507, 1719-1727 (Dec. 2009).

[3] Dravins, D., Lagadec, T., and Nuñez, P. D., "Stellar Intensity Interferometry over Kilometer Baselines: Optical aperture synthesis with electronically connected telescopes," IAU General Assembly 22, 2233727 (Aug. 2015).

[4] Stipčević, M., Skenderović, H., and Gracin, D., "Characterization of a novel avalanche photodiode for single photon detection in VIS-NIR range," Optics Express 18, 17448 (Aug. 2010).

[5] Zhu, D., Choi, H., Lu, T.-J., Zhao, Q., Dane, A., Najafi, F., Englund, D. R., and Berggren, K., "Superconducting nanowire single-photon detector on aluminum nitride," in [Conference on Lasers and Electro-Optics], Conference on Lasers and Electro-Optics, FTu4C.1, Optical Society of America (2016).

[6] Natarajan, C. M., Tanner, M. G., and Hadfield, R. H., "Superconducting nanowire single-photon detectors: physics and applications," Superconductor Science Technology 25, 063001 (June 2012).

[7] Tektronix, "Tektronix FCA3000 and FCA3100 Series Datasheet." Tektronix, 25 July 2015 http://www.tek. com/sites/tek.com/files/media/media/resources/FCA3000_Series_Timer-Counter-Analyzers_ Datasheet_3CW-25556-4_1.pdf. (Accessed: 5 July 2016).

[8] Voxtel, "YVX-657 Multi-channel TDC." Voxtel, 5 July 2016 http://voxtel-inc.com/files/2013/04/ TDC-VYX-657-datasheet.pdf. (Accessed: 5 July 2016).

[9] CASPER, "CASPER-Collaboration for Astronomy Signal Processing and Electronics Research." CASPER group, 2009 https://casper.berkeley.edu/. (Accessed: 5 July 2016).

[10] CASPER, "ROACH2 Revision 2 CASPER." CASPER group, 11 October 2013 https : //casper . berkeley . edu/wiki/ROACH-2_Revision_2. (Accessed: 5 July 2016).

[11] Excelitas, "SPCM-AQ4C Single Photon Counting Module 4 Channel Array Datasheet." Excelitas Technologies, Sept 2014 http://www.excelitas.com/downloads/DTS_SPCM-AQ4C.pdf. (Accessed: 5 July 2016).

[12] Pilyavsky, G., Mauskopf, P., Smith, N., Schroeder, E., Sinclair, A., van Belle, G., Hinkel, N., and Scowen, P., "SINGLE PHOTON INTENSITY INTERFEROMETRY (SPIIFY): UTILIZING AVAILABLE TELESCOPES," in prep. (2016).

[13] Bessell, M. S., "UBVRI photometry. II - The Cousins VRI system, its temperature and absolute flux calibration, and relevance for two-dimensional photometry," pasp 91, 589-607 (Oct. 1979).

[14] Campins, H., Reike, G. H., and Lebofsky, M. J., "Absolute Calibration of Photometry at 1 through $5 \mu \mathrm{m}$," in [Bulletin of the American Astronomical Society], baas 16, 941 (Sept. 1984).

[15] Schneider, D. P., Gunn, J. E., and Hoessel, J. G., "CCD photometry of Abell clusters. I - Magnitudes and redshifts for 84 brightest cluster galaxies," apj 264, 337-355 (Jan. 1983). 